Trauma Berufskrankh 2015 • 17[Suppl 1]:148-156 DOI 10.1007/s10039-013-1984-z

Online publiziert: 14. September 2013

(c) Springer-Verlag Berlin Heidelberg 2013

R. Ketterl · S. Ewender

Schwerpunkt Hand- und Wirbelsäulenchirurgie, Unfallchirurgie und orthopädische Chirurgie,

Klinikum Traunstein, Kliniken Südostbayern AG, Traunstein

\title{
Behandlungsoptionen bei Verletzungen der unteren Halswirbelsäule
}

lung von ossären und ligamentären Verletzungen der Halswirbelsäule berücksichtigt werden.

Nach Durchführung einer strukturierten umfassenden klinischen Untersuchung, einschließlich der Erhebung eines exakten neurologischen Status, muss die vorliegende Verletzung unter Berücksichtigung einer zielgerichteten bildgebenden Diagnostik differenziert bewertet werden. Stabile Verletzungen können einer konservativen Behandlung zugeführt werden, während bei instabilen Verletzungen in der Regel nur eine operative Versorgung in Betracht kommt [8].

Ziel des folgenden Beitrags ist, eine Behandlungsempfehlung anhand aktueller Arbeiten und der eigenen Erfahrungen mit Betroffenen zu geben.

\section{Patienten und Methodik}

In einem 10-Jahres-Zeitraum von 20032012 wurden in unserem Klinikum 142 Patienten, 53 Frauen und 89 Männer mit einem Durchschnittsalter von 43 Jahren, mit instabilen Verletzungen im Bereich der unteren Halswirbelsäule operativ versorgt.

\section{Diagnose}

\section{Klinische Untersuchung}

Die im Rahmen der Inspektion, Palpation und Erhebung des neurologischen Status gewonnenen Daten müssen analy- siert werden. Abklärungspflichtig sind dabei v. a.

- ein spontaner Nackenschmerz,

- eine schmerzhafte Palpation,

- das Vorhandensein von Bewegungsschmerzen,

- eine Haltungsinsuffizienz sowie

- die Angabe von Schluckbeschwerden.

Ergeben sich bei der neurologischen Statuserhebung Auffälligkeiten, ist auch bei einem bereits diskreten Befund eine umfassende weitere bildgebende Abklärung erforderlich.

\section{Bildgebende Diagnostik}

Im Bereich der unteren Halswirbelsäule ist die Durchführung einer konventionellen Röntgenaufnahme obligat. Ergeben sich bereits bei dieser Untersuchung Instabilitätszeichen wie eine segmentale Verschiebung, Kyphosierung, erweiterter Bandscheibenraum, Luxation oder Subluxation der Facettengelenke oder eine Inkongruenz der lordotischen Hilfslinien, ist eine weitere Schnittbilddiagnostik mittels Computertomographie (CT) mit multiplanaren Rekonstruktionen angezeigt. Auch bei einem durch konventionelle Aufnahmen schwer zu beurteilenden zervikothorakalen Übergang ist die CT-Diagnostik erforderlich.

Die Durchführung von Flexions- und Extensionsaufnahmen unter Bildwandlerkontrolle oder einer Magnetresonanztomographie (MRT) ist indiziert beim Verdacht auf eine diskoligamentäre Instabi- 
lität oder beim Nachweis eines neurologischen Defizits ohne erkennbare Verletzung auf den konventionellen oder CTAufnahmen $[8,22]$. Weiterhin sind diese Untersuchungen bei einem bewusstlosen Patienten mit adäquatem Unfallmechanismus und beim polytraumatisierten Patienten zu empfehlen.

\section{Unfallursache}

Häufigste Unfallursache ist der Verkehrsunfall, gefolgt vom Sturz aus großer Höhe, Sport- sowie Zweiradunfällen [19]. Von einer ähnlichen Verteilung berichtete Bühren [5] in einer Darstellung der Ergebnisse der Arbeitsgemeinschaft für Wirbelsäulenchirurgie der DGU (Deutsche Gesellschaft für Unfallchirurgie). Hierin enthalten war jedoch ein Anteil von 21\% mit banalen Unfallereignissen bei geriatrischen Patienten [5].

In unserem eigenen Krankheitsgut der letzten 10 Jahre waren mehr als $40 \%$ der Verletzungen durch Verkehrsunfälle verursacht. Aufgrund eines hohen Sport- und Freizeitwerts unserer Region ist der Anteil durch sportliche Aktivitäten verursachter Verletzungen der unteren Halswirbelsäule mit annährend 1/4 der Fälle hoch. Verletzungen durch Sturz aus großer Höhe oder im Rahmen eines Bagatelltraumas sind in unserem Krankengut zahlenmäßig gleich häufig vertreten ( $\bullet$ Abb. 1).

\section{Altersverteilung}

Verletzungen im Bereich der unteren Halswirbelsäule treten am häufigsten in den Altergruppen der 20- bis 29-Jährigen und der 30- bis 39-Jährigen auf. Alle übrigen Altergruppen sind zahlenmäßig annährend gleich betroffen bis auf die Altersgruppe der unter 10-Jährigen

(- Abb. 2).

In Abb. 3 ist dargestellt, welcher Anteil der Patienten zum Zeitpunkt des Unfalls wesentliche Wirbelsäulenveränderungen aufwies. Veränderungen im Sinne einer fortgeschrittenen Degeneration oder einer Spinalkanalstenose fanden sich bei $11 \%$ unserer Patienten, bei 2 Patienten lag eine instabile Verletzung bei M. Bechterew vor.

Trauma Berufskrankh 2015 • 17[Suppl 1]:148-156 DOI 10.1007/s10039-013-1984-Z

(c) Springer-Verlag Berlin Heidelberg 2013

\section{R. Ketterl · S. Ewender \\ Behandlungsoptionen bei Verletzungen der unteren Halswirbelsäule}

\section{Zusammenfassung}

Verletzungsursachen. Verletzungen im Bereich der unteren Halswirbelsäule (HWS) werden vorwiegend durch Verkehrs-, Sport- und Freizeitunfälle ausgelöst, können jedoch auch bei Bagatelltraumen entstehen.

Diagnostik. Verletzungen im Bereich der unteren HWS erfordern eine exakte klinische und radiologische Abklärung. Neben einer konventionellen radiologischen Bildgebung sind Schnittbildverfahren wie Computer-(CT) oder Magnetresonanztomographie (MRT) großzügig anzuwenden.

Therapie. Die Therapieentscheidung wird von der vorliegenden Verletzungsmorphologie, dem Instabilitätsgrad, evtl. vorliegenden neurologischen Ausfällen, Begleitverletzungen und dem Allgemeinzustand des Verletzten bestimmt. Luxationen und Luxationsfrakturen müssen zeitnah reponiert werden. Die ventrale interkorporelle Spondylodese ist das Operationsverfahren der Wahl. Sie erfolgt mittels Knochenblock, der zur Vermeidung einer Entnahmemorbidität zunehmend durch Titancages ersetzt wird, und Anbrin- gung einer winkelstabilen Platte. Zur Dekompression dorsaler Strukturen sowie bei Neuroforameneinengung und epiduralem Hämatom ist die dorsale Versorgung erforderlich. Eine dorsoventrale Spondylodese ist selten indiziert und wird bei M. Bechterew, nicht reponierbaren und hochinstabilen Verletzungen ausgeführt.

Outcome. Die ventrale Fusion zeigt eine niedrige Komplikationsrate. Die funktionellen Einbußungen sind gering und werden subjektiv nicht als störend empfunden. Eine adäquate operative Versorgung führt zu einer geringen Schmerzintensität mit mehr als $70 \%$ völlig schmerzfreier Patienten. Das posttraumatische neurologische Defizit ist postoperativ in den meisten Fällen verbessert. Das Outcome wird jedoch häufig durch das initiale neurologische Defizit bestimmt.

\section{Schlüsselwörter}

Wirbelsäulenverletzungen · Operative Versorgung $\cdot$ Spondylodese $\cdot$ Neurologisches Defizit · Outcome

\section{Treatment options for injuries of the lower cervical spine}

\section{Abstract}

Causes. Injuries of the lower cervical spine are mainly caused by traffic, sport or recreational accidents. However they can also occur due to minor trauma.

Diagnosis. A precise clinical and radiological investigation is essential. In addition to conventional radiologic imaging, sectional imaging techniques like CT or MRI should be used generously.

Treatment. Criteria for treatment selection are the morphology of the injury, the degree of instability, the occurrence of neurological deficits, collateral injuries and the general state of health of the patient. Dislocations and luxation fractures need to be reduced as soon as possible. Spondylodesis is performed using a bone-block and a fixed-angle plate. Titanium cages instead of bone blocks are increasingly applied to prevent the morbidity caused by the bone harvesting. A dorsal approach is necessary for the decompression of dorsal structures as well as in case of compression of the neuroforamen or in epidural hematoma. A dorsoventral spondylodesis is rarely indicated but is required in Bechterew's disease, non-reducible or highly unstable injuries.

Outcome. Ventral fusion has the lowest rate of complications. The patients generally perceive functional deficits that occur as minor and not disturbing. Adequate surgical treatment leads to a completely pain-free state in $70 \%$ of patients. In most cases, the posttraumatic neurological deficit could be improved postoperatively. However the outcome of this procedure is often predefined by the initial neurologic deficit.

\section{Keywords}

Spinal injuries - Operative therapy .

Spondylodesis · Neurological deficit .

Treatment outcome 


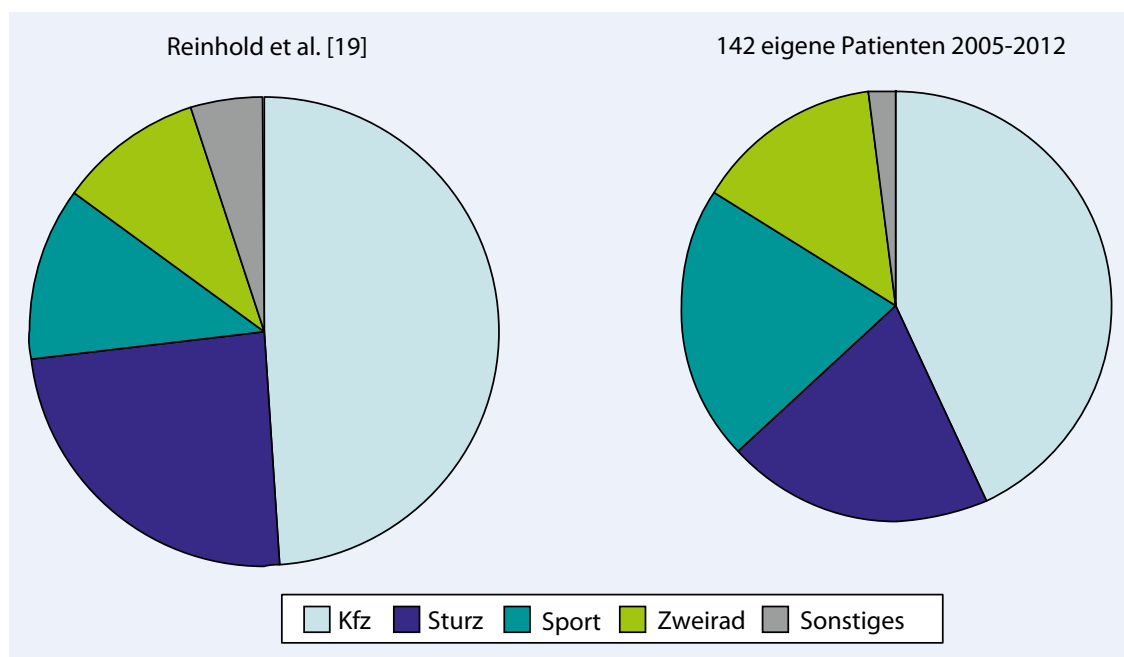

Abb. $1 \Delta$ Unfallursache, $K f z$ Kraftfahrzeug

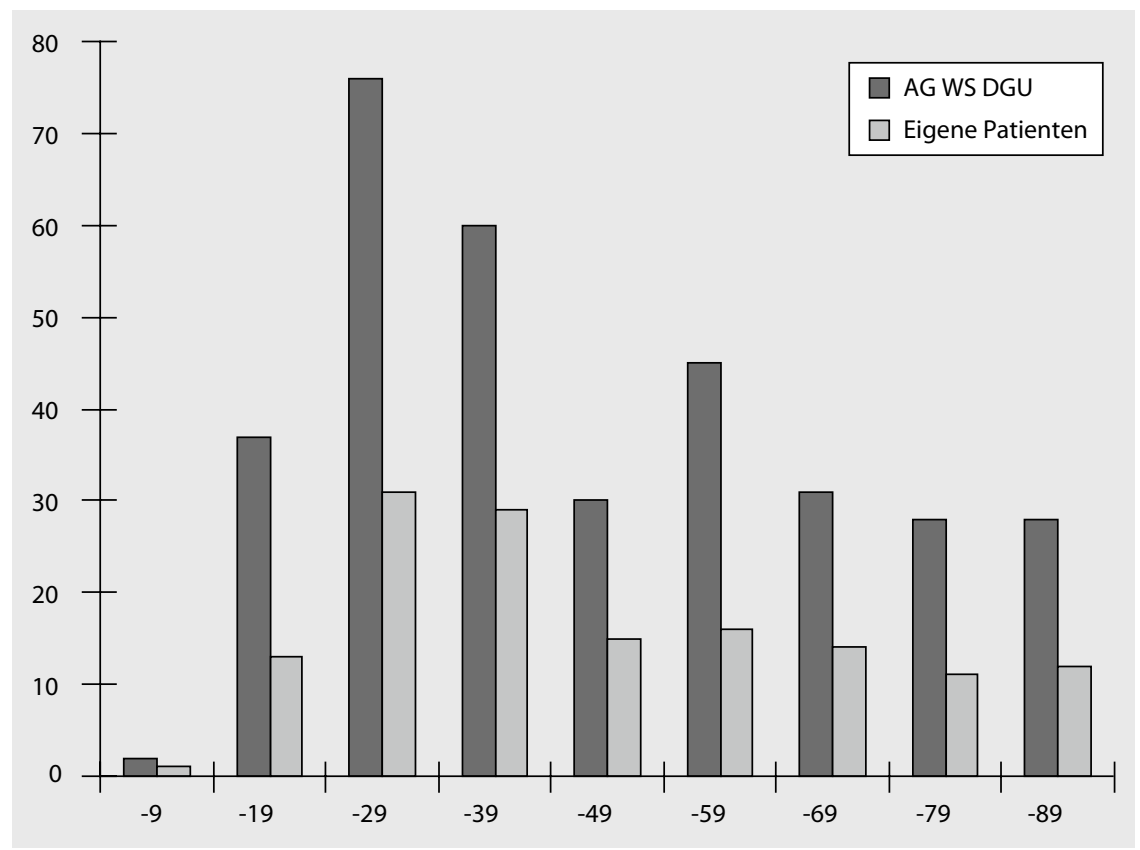

Abb. $2 \Delta$ Altersverteilung, AG WS DGU Arbeitsgemeinschaft für Wirbelsäulenchirurgie der DGU (Deutsche Gesellschaft für Unfallchirurgie)

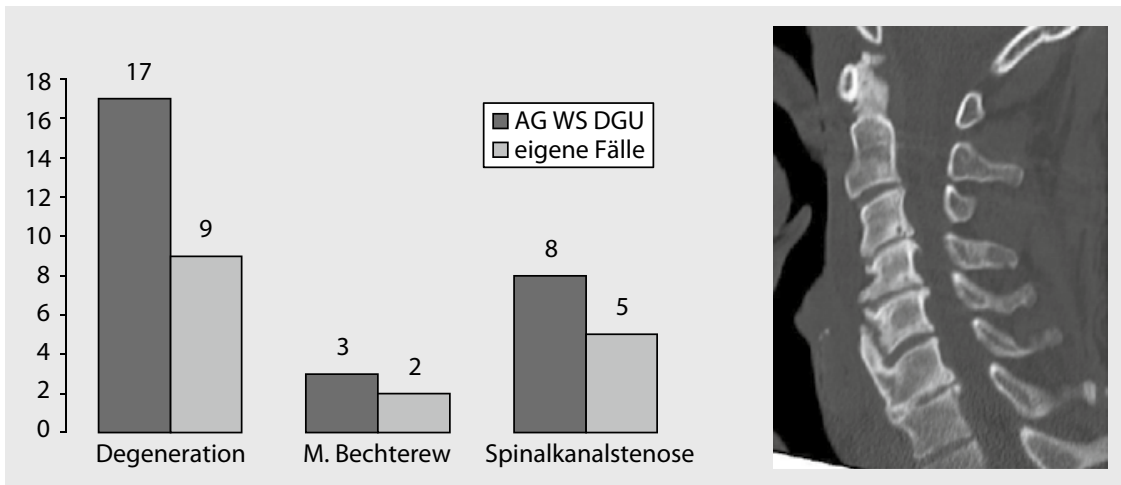

Abb. $3 \boldsymbol{\Delta}$ Vorerkrankungen, AG WS DGU Arbeitsgemeinschaft für Wirbelsäulenchirurgie der DGU (Deutsche Gesellschaft für Unfallchirurgie)

\section{Ergebnisse}

\section{Verletzungsarten}

Die verschiedenen Verletzungstypen können im Bereich der unteren HWS entsprechend der AO-Klassifikation (AO: Arbeitsgemeinschaft für Osteosynthesefragen; [15]) eingeteilt werden. Wir verwendeten diese Zuordnung modifiziert nach den Vorgaben von Ulrich u. Bühren [21]. Aus - Abb. 4 ist die Verteilung der spezifischen Verletzungsformen an der unteren Halswirbelsäule in unserem Patientengut ersichtlich, am häufigsten waren A-Verletzungen, gefolgt von B-Verletzungen und in einem geringeren Anteil CVerletzungen.

Die Verletzungshöhe betraf überwiegend die beiden Segmente C5/C6 und C6/C7 (C: zervikal), die exakte Zuordnung der Patienten ist $\mathbf{A b b .} 5$ zu entnehmen. Eine Verletzung auf mehreren Etagen fand sich bei 17 Patienten.

\section{Therapie}

Typische Versorgungsbeispiele sind in den Fallberichten 1-4 dargestellt.

Fallbeispiel 1. Der 48-jährige Patient wurde bei einem Segelfliegerabsturz schwer verletzt. Bei der primären Diagnostik mittels CT im Rahmen der Polytraumaspirale wurde eine verhakte $\mathrm{Lu}$ xation beidseits im Segment C5/C6 diagnostiziert (- Abb. 6a). Nach geschlossener Reposition konnte in der Abklärung mittels MRT die traumabedingte Myelopathie im Segment C5/C6 dargestellt werden (- Abb. 6b). Die operative Versorgung erfolgte über einen ventralen $\mathrm{Zu}$ gang mit Ausräumung des Bandscheibenfachs $\mathrm{C} 5 / \mathrm{C} 6$ und monosegmentaler ventraler Fusion mit Bandscheibencage und winkelstabiler Platte (• Abb. 6c-f). Die primären neurologischen Störungen besserten sich zunehmend und waren zum Zeitpunkt der Nachuntersuchung nach 2 Jahren völlig verschwunden.

Fallbeispiel 2. Die einseitig verhakte Luxation C5/C6 im Rahmen eines Sportunfalls beim Mountainbiking wurde operativ mit ventralem Zugang und Reposition der Luxation sowie monosegmenta- 


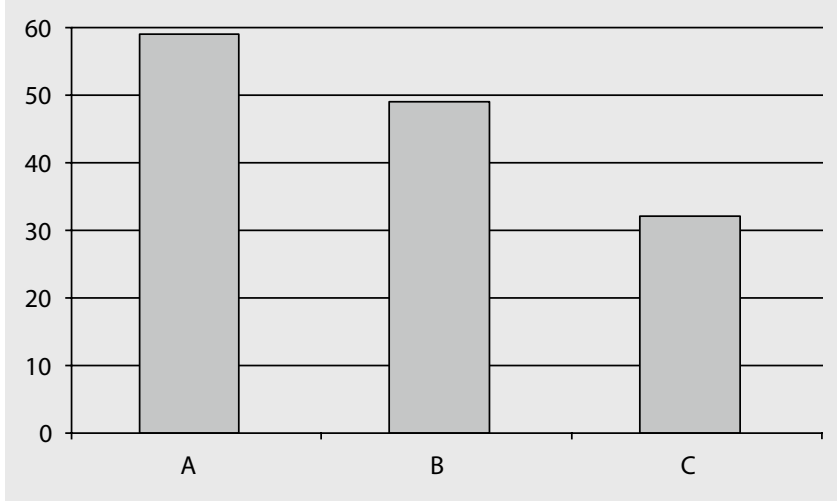

Abb. $4 \Delta$ \ Verletzungsklassifikation

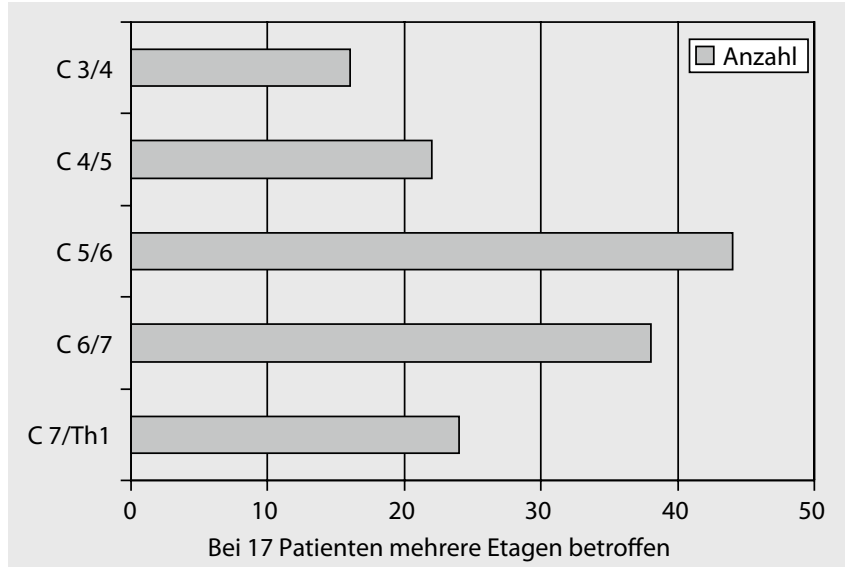

Abb. $5 \Delta$ Verletzungshöhe, C zervikal, Th thorakal
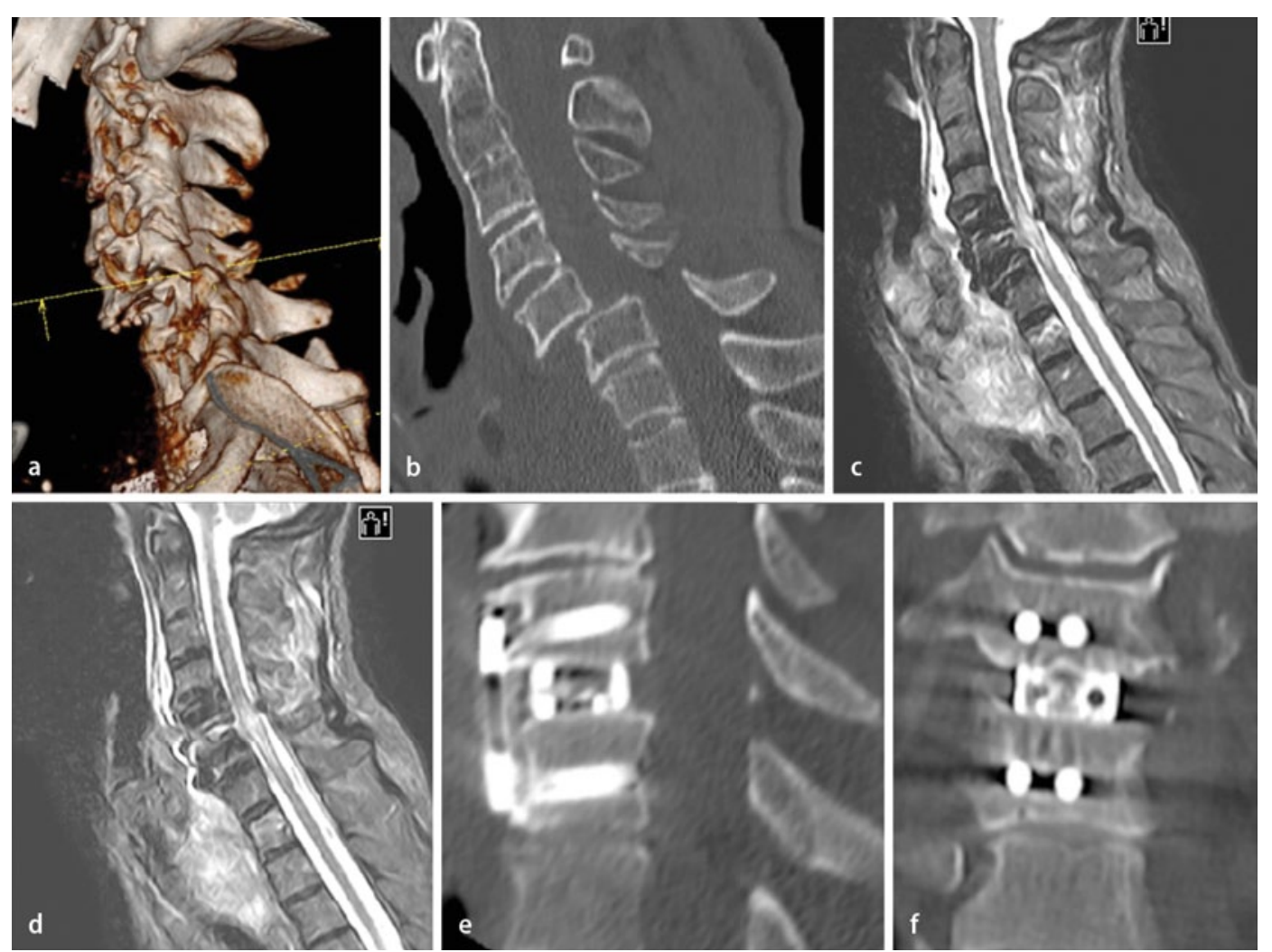

Abb. 64 Fallbeispiel 1: verhakte Luxation $\mathrm{C} 5 / \mathrm{C} 6$, a primäre Diagnostik mittels CT, b MRT nach geschlossener Reposition: traumabedingte Myelopathie im Segment C5/C6, c-f operative Versorgung mit Ausräumung des Bandscheibenfachs $\mathrm{C} 5 / \mathrm{C} 6$ und monosegmentaler ventraler Fusion, weitere Erläuterungen $s$. Text

ler Fusionierung mit Knochenblock und winkelstabiler Platte versorgt (• Abb. 7).

Fallbeispiel 3. Die komplexe HWK7Berstungsfraktur (HWK: Halswirbelkörper) nach Treppensturz wurde über einen ventralen Zugang mittels Vertebrektomie C7 mit ventraler Dekompression des Spinalkanals und Interposition eines SynMesh - Wirbelkörperersatzes sowie ventraler Verplattung C6-Th1 (Th: thorakal) versorgt (• Abb. 8).
Fallbeispiel 4. In den primären Röntgenaufnahmen stellte sich der zervikothorakale Übergang nur ungenügend dar. Mit der sich anschließenden CT-Diagnostik (- Abb. 9a,b) konnte das Ausmaß der vorliegenden Verletzung mit einer beidseits verhakten Luxation C7/Th1 dokumentiert werden. Sie ließ sich nicht geschlossen reponieren. Wegen der zusätzlich bestehenden Radikulopathie erfolgte zunächst eine dorsale Repositionierung mit Dekompression der Nervenwurzel C7 und dorsaler Stabilisierung mittels Cervi- fix $^{\oplus}$ (- Abb. 9c,d). Nach Umlagern wurde die Versorgung mit Ausräumung der Bandscheibe C7/Th1, Interposition eines Knochenblocks und Einbringen einer ventralen winkelstabilen Platte komplettiert (• Abb. 9e,f). Die primäre neurologische Störung war im weiteren Verlauf rückläufig und zum Zeitpunkt der Nachuntersuchung nicht mehr nachweisbar.

In der überwiegenden Anzahl der Fälle erfolgte eine monosegmentale interkorporelle Fusion. Bei 30 Patienten war eine bisegmentale Fusionierung erforderlich. 


\section{Wirbelsäulenverletzungen}
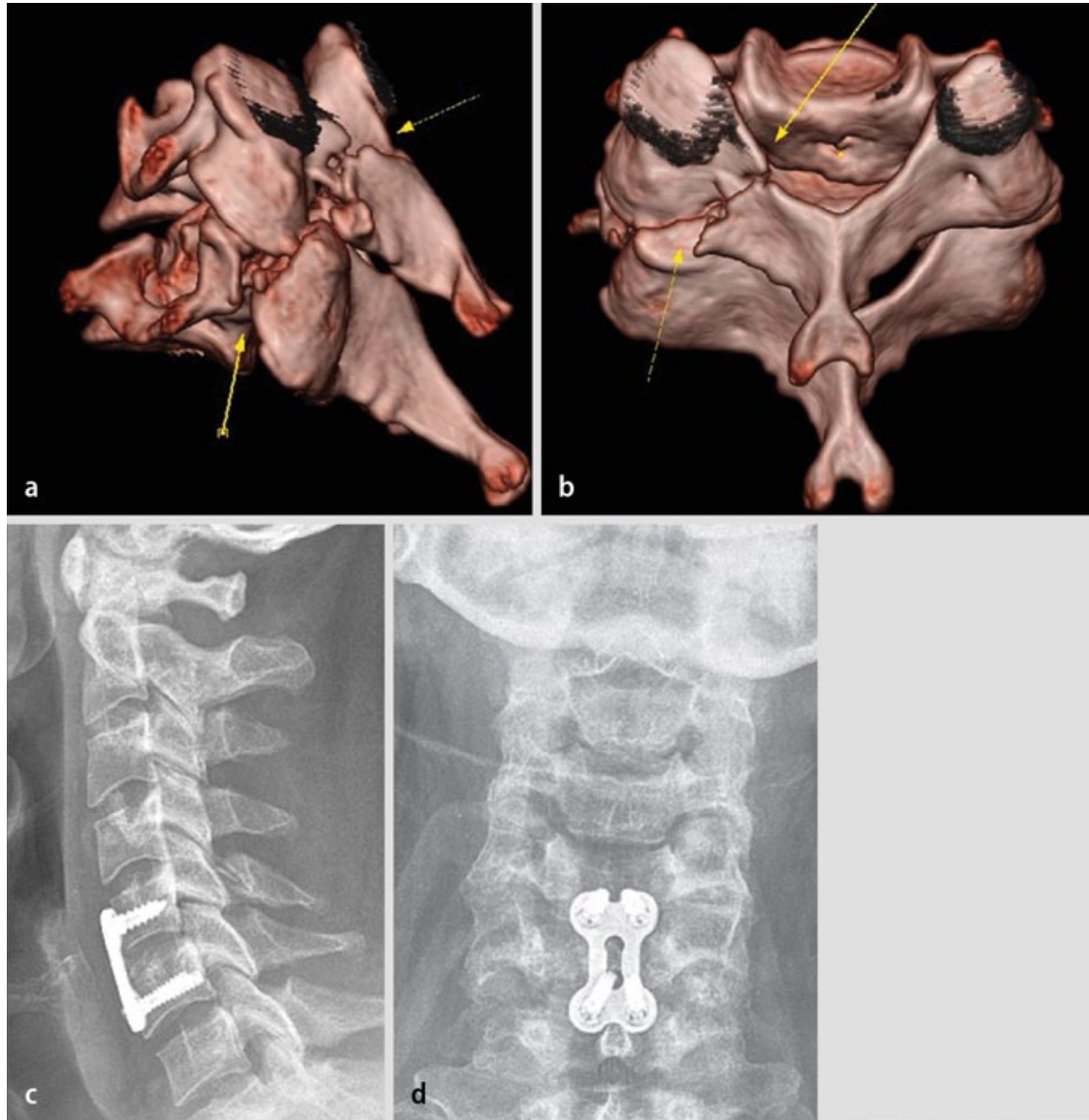

Abb. 74 Fallbeispiel 2: a,b einseitig verhakte Luxation $\mathrm{C} 5 / \mathrm{C} 6$, c,d operative Versorgung, weitere Erläuterungen $\mathrm{s}$. Text
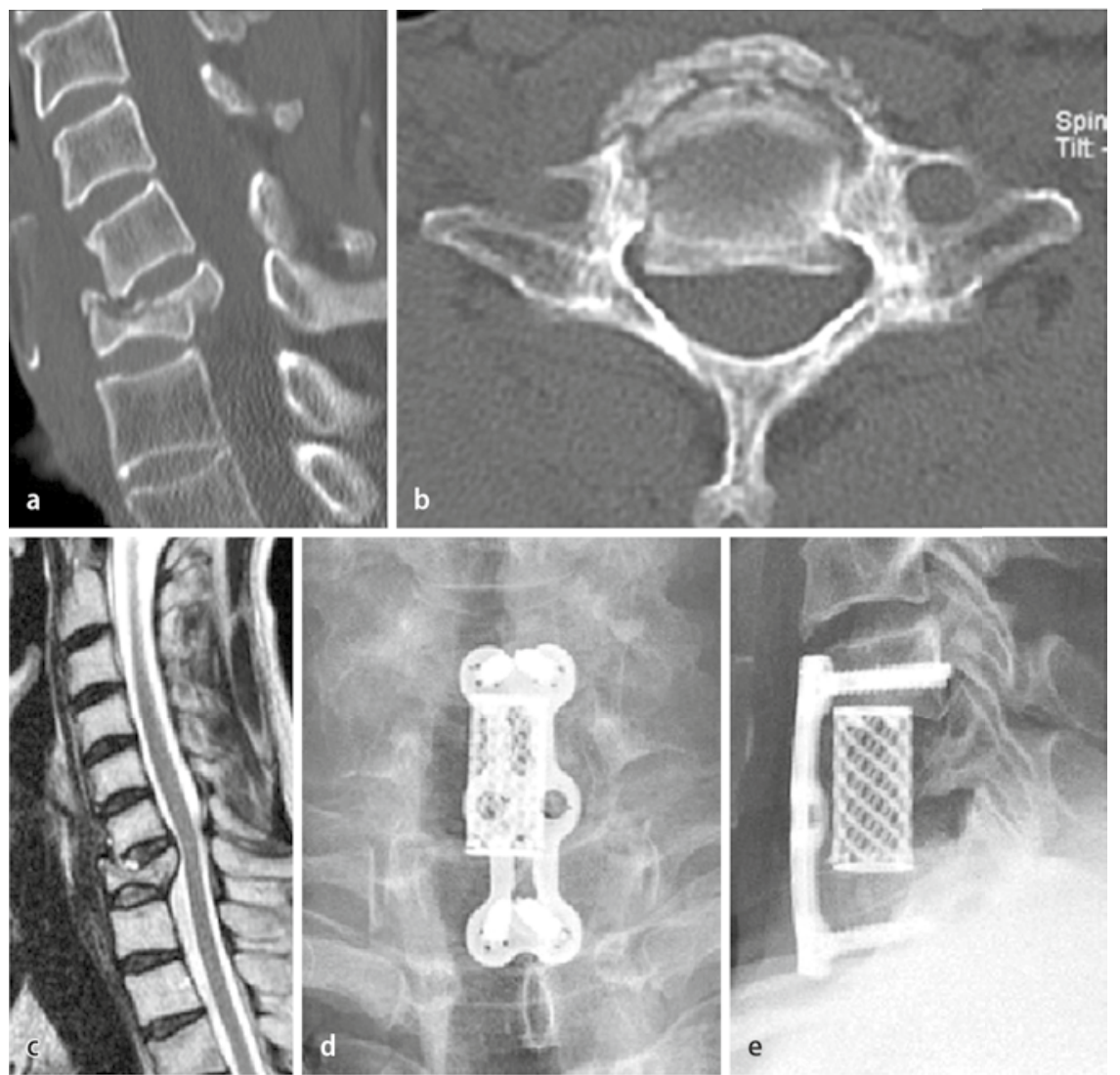

Abb. 84 Fallbeispiel 3: a,b komplexe Berstungsfraktur des Halswirbelkörpers 7, c-d operative Versorgung, weitere Erläuterungen s. Text 

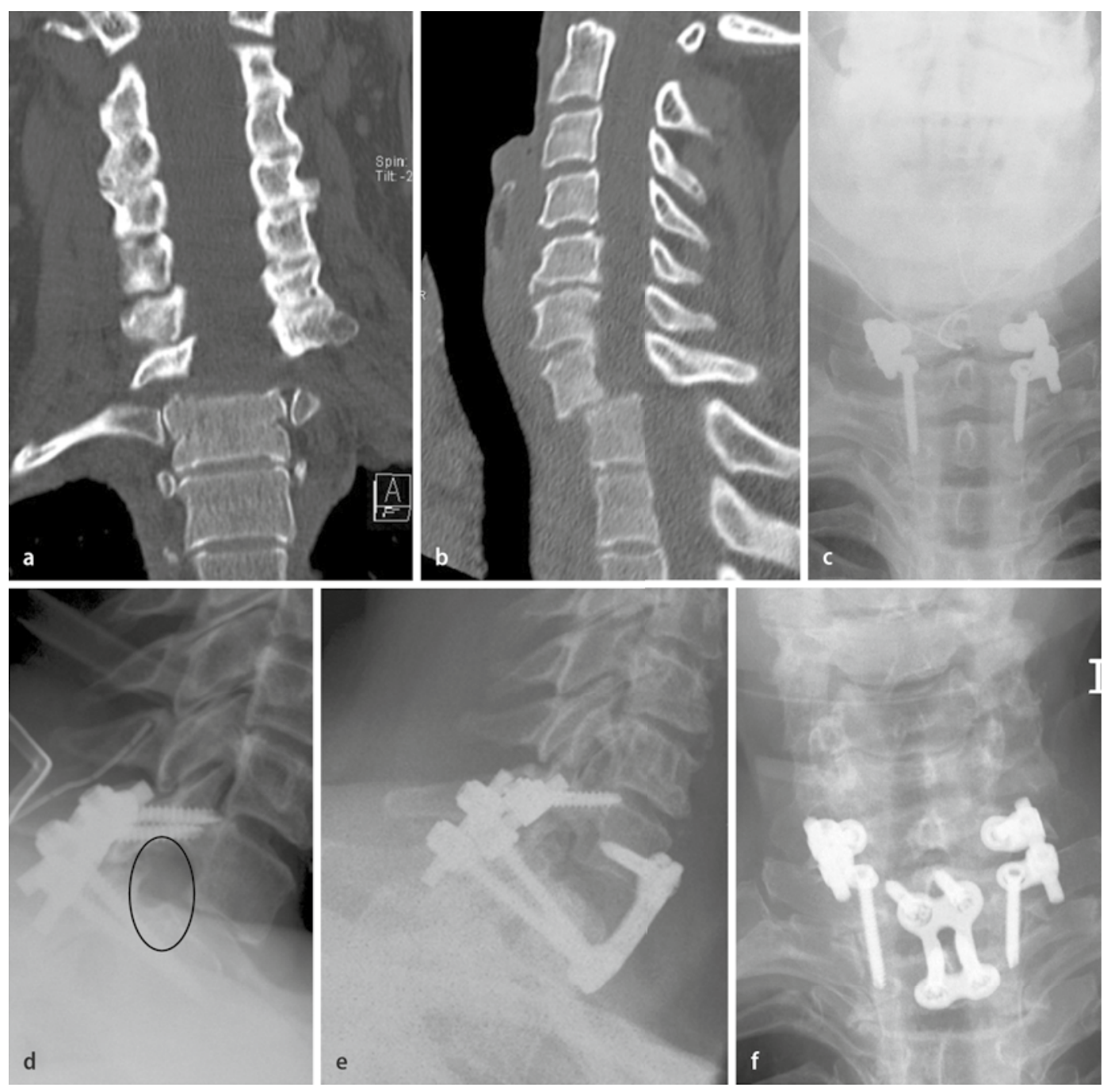

Abb. 9 Fallbeispiel 4: beidseits verhakte Luxation C7/Th1 mit Radikulopathie, weitere Erläuterungen s. Text
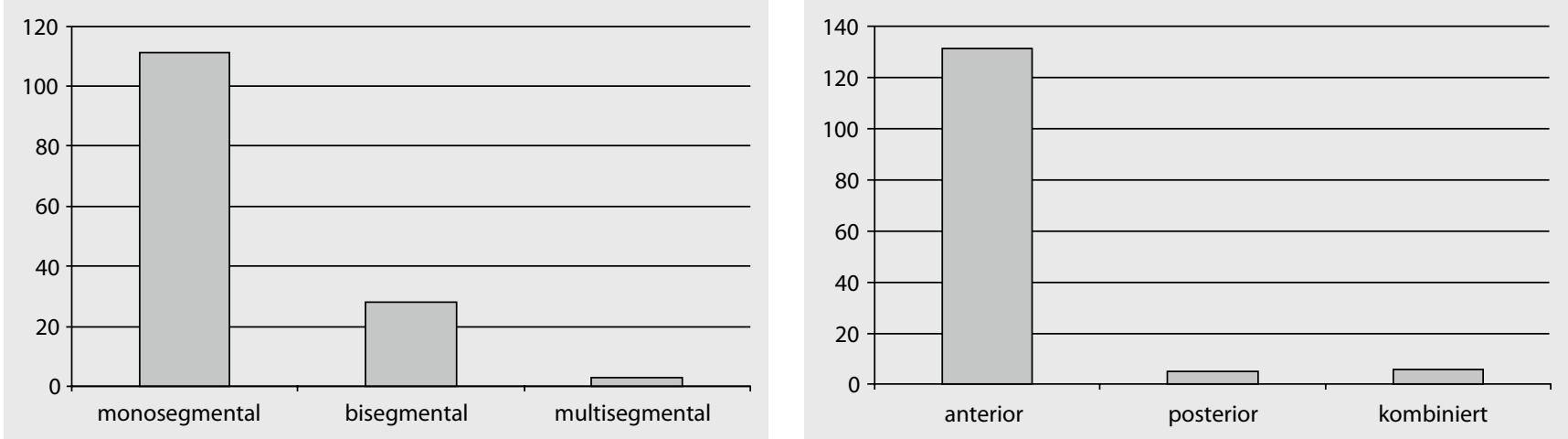

Abb. $10 \Delta$ Anzahl versorgter Segmente

Abb. $11 \Delta$ Art des Zugangswegs

In 4 Fällen mit einer Mehretagenverletzung wurden 3 und mehr Bewegungssegmente fusioniert ( $\bullet$ Abb. 10).

In 131 Fällen wurde rein ventral vorgegangen. Bei 5 Patienten war ein dorsaler Zugang zur Dekompression und Stabilisierung ausreichend. In 6 Fällen wur- de ein kombiniertes dorsoventrales Vorgehen erforderlich (• Abb. 11).

\section{Outcome}

Bezüglich des neurologischen Status lag zum Zeitpunkt des Unfalls bei 20 Patien- ten eine komplette Querschnittsymptomatik vor. In der postoperativen Phase waren noch 18 Patienten von einer solchen betroffen. Im Rahmen der Nachuntersuchung von 128 Patienten bestand eine komplette Querschnittsymptomatik unverändert bei 12 Patienten. Insgesamt 


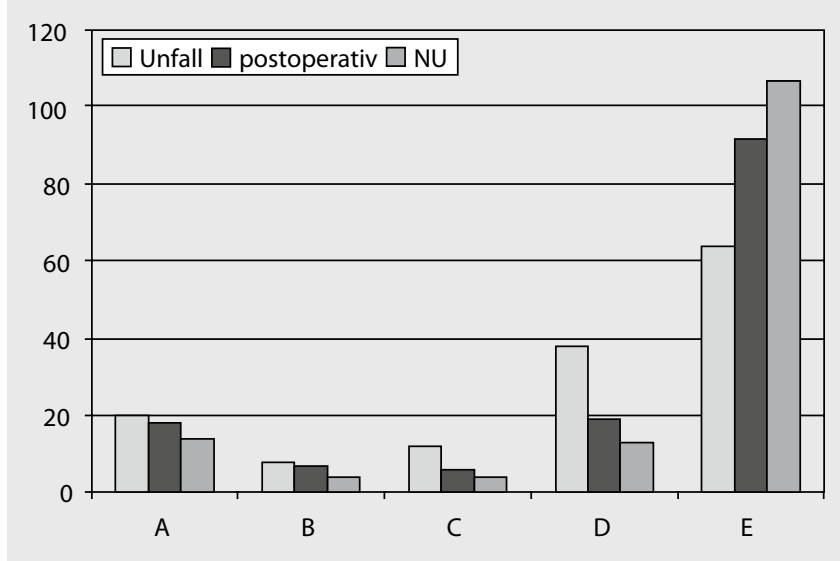

Abb. $12 \Delta$ Neurologischer Status, NU Nachuntersuchung, ASIA: A kompletter Querschnitt, $B$ inkompletter Querschnitt, sensible Funktionen erhalten, $C$ inkompletter Querschnitt, motorische Funktionen erhalten, $D$ inkompletter Querschnitt, Kennmuskeln mit Kraftgrad $\geq 3, E$ normale sensible und motorische Funktion

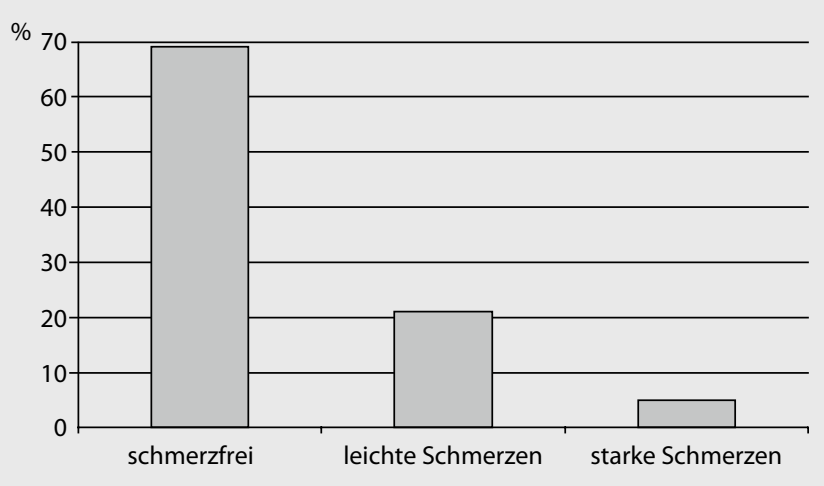

Abb. $13<$ Schmerzen

zeigte sich eine Verbesserung des neurologischen Status, sodass sich im Rahmen der Nachuntersuchung bei 98 der 128 Patienten ein vollständig unauffälliger neurologischer Befund ergab ( $\bullet$ Abb. 12).

Die Komplikationsrate bei unseren Patienten war gering: Es traten je eine Infektion beim ventralen (entspricht 0,7\%) und beim dorsalen Zugangsweg (entspricht 9,9\%) auf. Eine fehlende knöcherne Überbrückung des eingebrachten Knochenspans und einen Korrekturverlust beobachteten wir jeweils in 1 Fall (• Tab. 1).

Im Rahmen der Nachuntersuchung zeigte sich bei mehr als 70\% der Patienten ein schmerzfreier Zustand. Leichte Schmerzen gaben $22 \%$ der Patienten an, lediglich $6 \%$ klagten über stärkere Schmerzen (• Abb. 13).

\section{Diskussion}

\section{Therapieentscheid}

Bei Verletzungen im Bereich der unteren Halswirbelsäule richtet sich das therapeutische Vorgehen nach der vorliegenden Verletzungsmorphologie, dem Instabilitätsgrad, evtl. vorliegenden neurologischen Ausfällen, Begleitverletzungen und dem Allgemeinzustand des Verletzten. Liegen stabile Verletzungen ohne neurologische Ausfälle vor, werden diese konservativ behandelt. Hierbei kommen rein funktionelle Behandlungen sowie externe Ruhigstellung in einer HWS-Orthese in Frage $[5,19]$. Geeignete Verletzungen dafür sind Distorsionen im Bereich der Halswirbelsäule, Dornfortsatz-, Lamina- oder Gelenkfortsatzfrakturen jeweils ohne Instabilität und Verletzungen der Halswirbelsäule mit Nachweis einer neurologischen Störung, aber ohne Hin- weise für knöcherne oder diskoligamentäre Einengung ohne Kompromittierung des Myelons oder der Nervenwurzel. Instabilitäten werden einer operativen Versorgung zugeführt. Das Ausmaß der Instabilität kann dabei nach den von White u. Pajabi [23] vorgegebenen Kriterien eingeschätzt werden.

\section{Operatives Vorgehen}

Im Rahmen einer operativen Behandlung müssen folgende Therapieziele erreicht werden.

- Dekompression nervaler Strukturen zur Normalisierung der neurologischen Funktion,

- Korrektur der Fehlstellung zur Vermeidung einer posttraumatischen sekundären Fehlhaltung,

- Wiederherstellung einer dauerhaften Stabilität zur Vermeidung chronischer Instabilitäten sowie

- Erhaltung von Bewegungssegmenten mit dem Ziel einer kurzstreckigen Fusion, um so die Überlastung von Nachbarsegmenten zu verhindern.

Liegen Luxationen oder Luxationsfrakturen vor, ist eine zeitnahe Reposition durchzuführen, da der Repositionserfolg und das Ausmaß der neurologischen Erholung vom Zeitintervall zwischen dem Unfall und der Reposition abhängen. Entsprechende Studien ergaben, dass die Reposition zu einem frühen Zeitpunkt besser gelingt und zudem zu besseren klinischen Ergebnissen führt [1, 4, 14, 16].

Handelt es sich um eine instabile Verletzung mit gleichzeitigem Vorliegen einer neurologischen Störung oder eine anhand der bildgebenden Diagnostik anzunehmende Kompression neurologischer Strukturen, ist eine notfallmäßige Operation mit Dekompression der nervalen Strukturen und Fusion der instabilen Läsion indiziert. Eine schnellstmögliche Dekompression führt dabei zu einem besseren Outcome [18]. Zudem kann durch eine sofortige operative Versorgung eine durch eine fortbestehende Instabilität verursachte Verschlechterung des neurologischen Befunds verhindert werden [3].

Lässt sich nach einer erfolgreichen geschlossenen Reposition in der Akutversorgung eine Spinalkanaleinengung nicht 


\begin{tabular}{|lll}
\hline Tab. 1 Komplikationsrate & \\
\hline Komplikation & $\begin{array}{l}\text { Anzahl } \\
\text { Patienten }\end{array}$ & $\begin{array}{l}\text { Anteil } \\
\text { (\%) }\end{array}$ \\
\hline Blutung & 2 & 1,4 \\
\hline Infektion ventral & 1 & 0,7 \\
\hline Infektion dorsal & 1 & 9,9 \\
\hline $\begin{array}{l}\text { Schluck- } \\
\text { beschwerden }\end{array}$ & 4 & 2,8 \\
\hline $\begin{array}{l}\text { Rekurrensparese, } \\
\text { reversibel }\end{array}$ & 2 & 1,4 \\
\hline Radikulopathie & 2 & 1,4 \\
\hline
\end{tabular}

mehr nachweisen oder besteht eine Einengung des Spinalkanals ohne neurologisches Defizit, kann eine endgültige operative Versorgung mit aufgeschobener Dringlichkeit elektiv geplant werden. Ähnlich verhält es sich bei Versorgungen einer Halswirbelsäulenverletzung im Rahmen eines Polytraumas [11].

Die operative Therapie der unteren Halswirbelsäule besteht in der Regel in einer rein ventralen Versorgung. Dies gilt sowohl für Verletzungen, die nur die vordere Säule betreffen, als auch für Verletzungen mit Verlagerung von Knochenund Bandscheibenanteilen in den Spinalkanal sowie Verletzungen der dorsalen Säule. Dabei wird neben der Ausräumung des Bandscheibenfachs, ggf. in Kombination mit einer ventralen Dekompression des Spinalkanals, eine interkorporelle ventrale Spondylodese durch Einbringen entweder eines Knochenblocks oder eines Titancages mit zusätzlicher Sicherung durch eine ventrale winkelstabile Platte durchgeführt. Bei Anwendung Letzterer reicht eine monokortikale Schraubeneinbringung in der Regel aus und ergibt eine vergleichbare Stabilität zur bikortikalen Schraubenplatzierung bei nichtwinkelstabiler Plattenanlage [6, 17]. Im Fall des Vorliegens eines osteoporotischen Knochens nutzen wir auch unter Verwendung einer winkelstabilen Platte eine bikortikale Schraubeneinbringung, um auf diese Weise einen Stabilitätsgewinn zu erzielen und eine zusätzliche dorsale Fixation zu vermeiden. Der Vorteil eines ventralen Zugangs besteht in dem geringen Weichteiltrauma und der günstigen Lagerung insbesondere beim polytraumatisierten Patienten und beim älteren Verletzten. Der Zugang zum Spinalkanal ist risikoärmer und zeigt eine gute Übersichtlichkeit bei der Notwendigkeit der Spinal- kanaldekompression zur Entfernung von verlagerten Diskusanteilen oder verlagerten Hinterkantenfragmenten $[9,19]$. Ein weiterer Vorteil des ventralen Zugangs ist die Schonung der nuchalen Muskeln. Die Operationstechnik einer ventralen interkorporellen Spondylodese sollte deshalb immer, wenn irgend möglich, durchgeführt werden. Die geringe Komplikationsrate beim ventralen Zugang zur Halswirbelsäule ist in der Regel mit einem vernachlässigbaren Blutverlust verbunden und zeichnet ihn somit als schnellen und schonenden Zugangsweg aus [2, 7, 8, 10, $12,20]$.

Die ventrale Spondylodese der unteren Halswirbelsäule ist als adäquates komplikationsarmes Operationsverfahren zur Versorgung eines großen Anteils instabiler Verletzungen zu anzusehen. Mit ihr wird eine hohe Rate an knöchernen Fusionen mit dauerhaft stabilen Verhältnissen erreicht, sie kann somit als das Mittel der Wahl bezeichnet werden $[2,7,10,12]$.

Die Notwendigkeit zur Verwendung des dorsalen Zugangs besteht bei erforderlicher Dekompression des Spinalkanals bei dorsal liegenden Einengungen oder bei Verlegung der Neuroforamina durch von dorsal her verlagerte Knochenanteile. Er ist ebenfalls erforderlich, wenn eine verhakte oder geschlossen nicht reponierbare Luxation oder Luxationsfraktur vorliegen. An dorsalen Stabilisationsverfahren kommt ein modulares Schraubenstabsystem mit Schraubenverankerung in der Massa lateralis oder transpedikulär in Frage. Ein rein dorsales Vorgehen kommt äußerst selten zur Anwendung. Häufig müssen die dorsalen Stabilisationsverfahren mit ventralen Fusionen kombiniert werden. Insbesondere gilt dies bei Vorliegen eines M. Bechterew. Hier empfiehlt sich die langstreckige dorsoventrale Versorgung zur Vermeidung eines Korrekturverlusts oder einer Schraubenlockerung bzw. eines Implantatbruchs $[9,13,24]$.

In der Diskussion entscheidend für die funktionellen Beeinträchtigungen im Rahmen von Halswirbelsäulenverletzungen ist der neurologische Status. Neurologische Störungen können sich vielgestaltig darstellen, von einfachen radikulären Störungen bis hin zu einer inkompletten oder kompletten Querschnittslähmung.
Durch eine adäquate und zeitgerechte Versorgung kann das primär vorliegende neurologische Defizit verbessert werden. Diese Aussage wird durch die Ergebnisse bei unseren Patienten und Berichte anderer Autoren [10] dokumentiert.

\section{Fazit für die Praxis}

- Das therapeutische Vorgehen bei Verletzungen im Bereich der unteren HWS hängt von der Verletzungsmorphologie, dem Instabilitätsgrad, evtl. neurologischen Ausfällen, Begleitverletzungen und dem Allgemeinzustand des Verletzten ab.

- Stabile Verletzungen ohne neurologische Ausfälle können konservativ behandelt werden, ansonsten sind operative Verfahren indiziert.

- Die ventrale Spondylodese der unteren Halswirbelsäule ist als adäquates komplikationsarmes Operationsverfahren zur Versorgung eines großen Anteils instabiler Verletzungen anzusehen und kann als das Mittel der Wahl bezeichnet werden.

- Ein rein dorsales Vorgehen kommt äußerst selten zur Anwendung. Eine Kombination der dorsalen Stabilisationsverfahren mit ventralen Fusionen ist ebenfalls nur selten erforderlich.

- Durch eine adäquate und zeitgerechte Versorgung kann das primär vorliegende neurologische Defizit verbessert werden.

\section{Korrespondenzadresse}

\section{Prof. Dr. R. Ketterl}

Schwerpunkt Hand- und Wirbelsäulenchirurgie, Unfallchirurgie und orthopädische Chirurgie, Klinikum Traunstein, Kliniken Südostbayern AG, Cuno-Niggl-Straße 3, 83278 Traunstein diana.stiller@klinikum-traunstein.de 


\section{Einhaltung ethischer Richtlinien}

Interessenkonflikt. R. Ketterl und S. Ewender geben an, dass kein Interessenkonflikt besteht.

Dieser Beitrag beinhaltet keine Studien an Menschen oder Tieren.

The supplement containing this article is not sponsored by industry.

\section{Literatur}

1. Aebi M, Mohler J, Zach GA, Morscher E (1986) Indication, surgical technique and results of 100 surgically-treated fractures and fracture-dislocations of the cervical spine. Clin Orthop Relat Res 203:244257

2. Blauth M, Schmidt U, Dienst M (1996) Langzeitergebnisse von 57 Patienten nach ventraler interkorporeller Spondylodese der unteren Halswirbelsäule. Unfallchirurg 99:925-939

3. Blauth M, Tscherne H (1998) Untere Halswirbelsäule. In: Tscherne H, Blauth M (Hrsg) Tscherne Unfallchirurgie. Springer, Berlin Heidelberg New York, S $153-240$

4. Brunette DD, Rockswold GL (1987) Neurologic recovery following rapid spinal realignment for com plete cervical spinal cord injury. J Trauma 27:445447

5. Bühren V (2002) Frakturen und Instabilitäten der Halswirbelsäule. Unfallchirurg 73:1049-1066

6. Daentzer D, Böker DK (2004) Operative Stabilisierungen traumatischer Instabilitäten der unteren Halswirbelsäule. Unfallchirurg 107:175-180

7. Garrey TA, Eismont FJ, Robert LJ (1992) Anterior decompression, structural bone grafting and Caspar plate stabilization for unstable cervical spine fractures and/or dislocations. Spine 17:431-435

8. Gonschorek O (2013) Frakturen der unteren Halswirbelsäule (C3-C7) In: Bühren V, Josten Ch (Hrsg) Chirurgie der verletzten Wirbelsäule. Springer, Berlin Heidelberg New York, S 182-195

9. Heineck J, Bergest $H$, Müller M et al (2007) Ventrale Versorgung einer Fraktur der Halswirbelsäule bei Morbus Bechterew und Struma permagna. Unfallchirurg 110:571-575

10. Hofmeister M, Bühren V (1999) Therapeutisches Konzept bei Verletzungen der unteren Halswirbelsäule. Orthopade 28:401-413

11. Ketterl R (2010) Vorgehen bei Wirbelsäulenverletzungen des polytraumatisierten Patienten. Trauma Berufskrankh [Suppl 2] 12:168-175

12. Kostaik JP, Conolly PJ, Esses SI, Suh P (1993) Anterior cervical plate fixation with the titanium hollow screw plate system. Spine 18:1273-1278

13. Lange U, Pape MC, Bastian L, Krettek C (2005) Management operativer Stabilisierungen von Halswirbelsäulenverletzungen bei Morbus Bechterew. Unfallchirurg 108:63-68

14. Lee AS, MacLean JCB, Newton DA (1994) Rapid traction for reduction of cervical spine dislocations. J Bone Joint Surg Br 76:352-356

15. Magerl F, Aebi M, Gertzbein SD et al (1994) A comprehensive classification of thoracic and lumbar injuries. Eur Spine J 3:184-201

16. Mirza SK, Krengel WF, Chapman JR (1999) Early versus delayed surgery for acute cervical spinal cord injury. Clin Orthop Relat Res 359:104-114
17. Northwang J, Ulrich C (2005) Operative Versorgung von HWS-Verletzungen Komplikationsmöglichkeiten. Trauma Berufskrankh [Suppl 2] 7:52635270

18. Papadopoulos SM, Selden WR, Quint DJ (2002) Immediate spinal cord decompression for cervical spinal cord injury; feasibility and outcome. JTrauma 52:323-332

19. Reinhold M, Blauth M, Rosiek R, Knop C (2006) Verletzungen der unteren Halswirbelsäule, Klassifikation und Behandlungskonzept. Unfallchirurg 109:471-482

20. Schleicher P, Scholz M, Schnake K, Kandziora F (2008) Standarddiagnostik und Managment von subaxialen HWS-Verletzungen. Trauma Berufskrankh [Suppl 2] 10:175-181

21. Ulrich C, Bühren V (2006) Verletzungen der Halswirbelsäule. Orthop Unfallchir Up2date 1:415-441

22. Vock B, Matschke $S$, Grützner PA, Wentzengen $A$ (2004) Erste Erfahrungen mit winkelstabilen Implantaten an der Halswirbelsäule. Trauma Berufskrankh [Suppl 1] 6:535-540

23. White AA, Pajabi MM (1990) Clinical biomechanics of the spine, 2. Aufl. Lippincott Williams \& Wilkins, Philadelphia

24. Zdichavsky M, Blauth M, Knop C et al (2005) Ankylosierende Spondylitis - Therapie und Komplikationen von 34 Wirbelsäulenfrakuren. Chirurg 76:967-975 